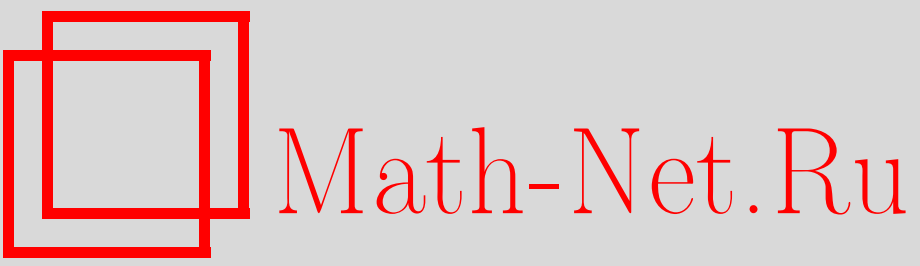

В. М. Бухштабер, Э. Г. Рис, Многозначные группы и $n$-алгебры Хопфа, УМH, 1996, том 51, выпуск 4, 149-150

DOI: https://doi.org/10.4213/rm998

Использование Общероссийского математического портала Math-Net.Ru подразумевает, что вы прочитали и согласны с пользовательским соглашением

http://www.mathnet.ru/rus/agreement

Параметры загрузки:

IP : 18.234 .197 .8

26 апреля 2023 г., 14:47:14 


\section{МНОГОЗНАЧНЫЕ ГРУППЫ И $n$-АЛГЕБРЫ ХОПФА}

\section{В. М. БУХштаБеР, Е. Г. РИС}

Заметка посвящена новым алгебраическим понятиям, которые возникли в ходе наших исследований по теории многозначных групп [1].

Пусть $(X)^{n}-n$-я симметрическая степень пространства $X$, т.е. фактор-пространство декартовой $n$-й степени $X^{n}$ по действию симметрической группы перестановок координат. Введем диагональное отображение $D=D_{n}: X \rightarrow(X)^{n}: x \rightarrow[x, \ldots, x]$ и отображение $1 \times D:(X)^{n} \times X \rightarrow(X \times X)^{n}:\left(\left[x_{1}, \ldots, x_{n}\right], x\right) \rightarrow\left[\left(x_{1}, x\right), \ldots,\left(x_{n}, x\right)\right]$. Аналогично вводится отображение $D \times 1: X \times(X)^{n} \rightarrow(X \times X)^{n}$.

ОПРеДЕЛЕНИЕ 1. $n$-значной группой называется пространство $X$ вместе с операциями умножения $\mu: X \times X \rightarrow(X)^{n}(\mu(x, y)=x \cdot y)$ и взятия обратного элемента $\tau: X \rightarrow X\left(\tau(x)=x^{*}\right)$, которые удовлетворяют аксиомам:

1) существует точка $e \in X$ (единица) такая, что $e \cdot x=x \cdot e=D(x)$;

2) отображения $(\mu)^{n}(1 \times D)(\mu \times 1)$ и $(\mu)^{n}(D \times 1)(1 \times \mu): X \times X \times X \rightarrow(X)^{n^{2}}$ совпадают (ассоциативность умножения);

3) $e \in\left(x \cdot x^{*}\right) \cap\left(x^{*} \cdot x\right)$.

Многозначная группа назьвается коммутативной, если $x \cdot y=y \cdot x$ для любых $x$ и $y$.

ОПРЕДЕЛЕНИЕ 2 . Пусть $n_{1}=n k$. Гомоморфизмом $n$-значной группы $\left(X, e_{X}, \mu_{X}, \tau_{X}\right)$ в $n_{1}$-значную группу $\left(Y, e_{Y}, \mu_{Y}, \tau_{Y}\right)$ называется отображение $f: X \rightarrow Y$ такое, что

$$
\left(f^{k} D_{k}\right)^{n} \mu_{X}=\mu_{Y}(f \times f) ; \quad f\left(e_{X}\right)=e_{Y} ; \quad f\left(\tau_{X}(x)\right)=\tau_{Y} f(x) .
$$

Ясно, что обычная (однозначная) группа $G$ с умножением $m$ является $n$-значной с умножением $\mu=D m$. Важный пример так называемых косетныx груnп возникает, когда мы рассматриваем группу $G$ вместе с некоторой подгруппой $A$ порядка $n$ группы автоморфизмов $\operatorname{Aut}(G)$.

Положим $X=G / A ; \mu(x, y)=\pi\left(m\left(\pi^{-1}(x), \pi^{-1}(y)\right)\right) ; \tau(x)=\pi\left(\tau_{G}\left(\pi^{-1}(x)\right)\right)$, где $\pi: G \rightarrow X$ - каноническая проекция и $\tau_{G}$ - взятие обратного в группе $G$. Непосредственная проверка показывает, что такие $X, \mu, \tau$ задают $n$-значную группу. Отметим (см. [1], [2]), что одна и таже $n$-значная группа может быть реализована как косетная для разных пар $\left(G_{1}, A_{1}\right)$ и $\left(G_{2}, A_{2}\right)$.

Пусть $f: X \rightarrow Y$ - некоторьй гомоморфизм $n$-значной группы $X$ в $m$-значную группу $Y$.

Положим $\operatorname{Ker} f=\left\{x \in X: f(x)=e_{Y}\right\}$. Тогда $\left(\operatorname{Ker} f, e_{X}, \mu_{X}, \tau_{X}\right)$ является $n$-значной подгруппой в $X$.

Обозначим через $\{r\}$ вычет целого числа $r$ по модулю $m$. Для множества $X$ из $m$ точек $\left(x_{0}, x_{1}, \ldots, x_{m-1}\right)$ положим $\mu\left(x_{p}, x_{q}\right) \in(X)^{2}$ равным $D\left(x_{p+q}\right)$, если $p+q<m$ и равным $\left[x_{p+q}, x_{p+q+1}\right]$, если $p+q \geqslant m$. Далее, положим $e=x_{0}, \tau\left(x_{0}\right)=x_{0}$ и $\tau\left(x_{k}\right)=x_{m-k}, k>0$. Непосредственная проверка показьвает, что такие операции $\mu, \tau$ и выбор единицы задают на $X$ структуру $(X, e, \mu, \tau)$ двузначной группы. В то же время, нетрудно показать [1], что эта двузначная группа не может быть реализована как косетная группа, если $m>2$.

Для коммутативной группы $G$ взятие обратного элемента является автоморфизмом, поэтому с каждой коммутативной группой канонически связана двузначная косетная группа. В случае формальных групп именно эта конструкция дала первые и важные в топологических приложениях двузначные формальные группы "квадрата модуля" (см. [3], [4]). В случае эллиптической кривой эта конструкция (см. [5]) приводит к структуре двузначной группы на комплексной проективной прямой $\mathbb{C P}(1)$. Отождествим $(\mathbb{C P}(1))^{2}$ с $\mathbb{C P}(2)$. Тогда справедлива следующая

Tеорема 3. Отображсение $\mu: \mathbb{C P}(1) \times \mathbb{C P}(1) \rightarrow \mathbb{C P}(2)$, которое в однородных координаmax имеет вид $\mu\left(\left(x_{0}: x_{1}\right),\left(y_{0}: y_{1}\right)\right)=\left(\left(x_{0} y_{0}-k^{2} x_{1} y_{1}\right)^{2}:-2\left(x_{0} y_{1}+x_{1} y_{0}\right)\left(x_{0} y_{0}+k^{2} x_{1} y_{1}\right)-\right.$ $\left.4 \delta x_{0} y_{0} x_{1} y_{1}:\left(x_{1} y_{0}-x_{0} y_{1}\right)^{2}\right)$, вместе с отображением $\tau\left(x_{0}: x_{1}\right)=\left(x_{0}: x_{1}\right)$ и единицей $e=(0: 1)$ задает структуру двузначной группь на $\mathbb{C P}(1)$ тогда и только тогда, когда $k\left(k-\delta^{2}\right) \neq 0$.

Пусть $k$-коммутативное кольцо с единицей 1 , содержащее $1 / n$. 
ОпредЕлЕниЕ 4 . $k$-линейное отображение $L: A \rightarrow B$ коммутативных $k$-алгебр называется

1) алгебраическим степени $n$ в $a \in A$, если существует полином

$p(a ; t)=t^{n}-\beta_{1}(a) t^{n-1}+\cdots+(-1)^{n} \beta_{n}(a) \in B[t]$ такой, что $\sum_{q \geqslant 0} \frac{L\left(a^{q}\right)}{t^{q+1}}=\frac{1}{n} \frac{d}{d t} \ln p(a ; t) ;$

2) $n$-алгебрачческим, если оно алгебраическое степени $n$ для всех $a \in A$.

В условиях определения 4 обозначим через $d_{L}\left(a_{1}, \ldots, a_{n+1}\right)$ определитель $(n+1) \times(n+1)$ матрицы $M_{L}\left(a_{1}, a_{2}, \ldots, a_{n+1}\right)=\left(m_{i j}\right)$, где $m_{i j}=0$, если $j>i+1 ; m_{i+1}=i ; m_{i i}=L\left(a_{i}\right)$ и $m_{i j}=L\left(a_{j} a_{j+1} \cdots a_{i}\right)$, если $j<i$.

Положим $R_{L}\left(a_{1}, a_{2}, \ldots, a_{n+1}\right)=\sum_{\sigma \in \Sigma_{n+1}} d_{L}\left(a_{\sigma(1)}, a_{\sigma(2)}, \ldots, a_{\sigma(n+1)}\right)$, где $\Sigma_{n+1}-$ симметрическая группа всех перестановок.

Теорема 5 . $k$-линейное отображение $L: A \rightarrow B$ коммутативных $k$-алгебр является $n$-алгебрачческим тогда и только тогда, когда $R_{L}\left(a_{1}, a_{2}, \ldots, a_{n+1}\right)=0$ для всех наборов $a_{1}, a_{2}, \ldots, a_{n+1}$.

СледСтвиЕ 6. Отображение $L$ тогда и только тогда является

1) 1-алгебрачческим, когда $L\left(a_{1} a_{2}\right)=L\left(a_{1}\right) L\left(a_{2}\right)$, т.е. $L$ - кольцевой гомоморфизм;

2) 2-алгебрачческим, когда $L\left(a_{1} a_{2} a_{3}\right)=L\left(a_{1}\right) L\left(a_{2} a_{3}\right)+L\left(a_{2}\right) L\left(a_{3} a_{1}\right)+L\left(a_{3}\right) L\left(a_{1} a_{2}\right)$ $-2 L\left(a_{1}\right) L\left(a_{2}\right) L\left(a_{3}\right)$.

ОПРЕДЕЛЕНИЕ 7. $n$-алгеброй Хопфа $(A ; m, \iota, \Delta, \varepsilon, s)$ называется $k$-линейное пространство $A$ вместе

1) со структурой коммутативной ассоциативной $k$-алгебры $(A ; m, \iota)$, где $m: A \otimes A \rightarrow A-$ умножение и $\iota: k \rightarrow A$ - единица;

2) со структурой коассоциативной коалгебры $(A, \Delta, \varepsilon)$, где $\Delta: A \rightarrow A \otimes A$ - диагональ и $\varepsilon: A \rightarrow k-$ коединица;

3) с кольцевым гомоморфизмом $\tau: A \rightarrow A$ (антиподом).

Более того, $\Delta$ является $n$-алгебраическим отображением таким, что полиномы $p(a ; t) \in$ $A \otimes A[t]$, существующие для каждого $a \in A$ (см. определение 4$),$ удовлетворяют следующим аксиомам:

1) $p(1 ; t)=(t-1 \otimes 1)^{n}$;

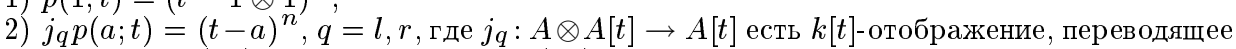
$a_{1} \otimes a_{2}$ в $\varepsilon\left(a_{1}\right) a_{2}$ при $q=l$ и в $a_{1} \varepsilon\left(a_{2}\right)$ при $q=r$

3) $m(1 \otimes s) p(a, \varepsilon(a))=m(s \otimes 1) p(a, \varepsilon(a))=0$. Хопфа.

ТеОрема 8. Пусть $X$ - некоторая $n$-значная группа. Тогда кольцо функций $F(X)$ u кольцо когомологий $H^{*}(X ; k)$ являются $n$-алгебрами Хопфа. (Здесь $k$-поле характеристики взаимно простой с $n$.)

Из следствия 6 и теоремы 8 вытекает, например,

СледСтвиЕ 9. На пространстве $\mathbb{C P}(m)$ при $m>1$ нельзя ввести структурь двузначной әруппьь.

Большая часть этих исследований была выполнена в течение визитов первого автора в Эдинбургский Университет благодаря EPSRC-Гранту.

\section{СПИСОК ЛИТЕРАТУРЫ}

[1] Buchstaber V.M., Rees E. G. Multivalued groups, their representations and Hopf algebras // Preprint. Univ. of Edinburgh, 1995. [2] Buchstaber V. M., Veselov A.P. // Internat. Math. Res. Notices. 1996. № 8. P. 381-400. [3] Бухштабер В. М., Новиков С. П. // Матем. сб. 1971. Т. 84. С. 81-118. [4] Бухштабер В. М. // Совр. пробл. матем. Т. 10. М.: ВИнИТИ, 1978. С. 5-178. [5] Бухштабер В. М. // УМН. 1990. Т. 45. № 3. С. 213-215.

Московский государственный университет им. М. В. Ломоносова; University of Edinburgh;

J.C. Maxwell Building
Принято редколлегией 17.07.1996 